\title{
Percepção das puérperas em relação ao atendimento recebido na unidade básica de saúde durante a consulta de pré-natal
}

\author{
Perception of puerperal women in relation to the care \\ received at the basic health unit during the prenatal consultation
}

Marcela Mika Kawatsu ${ }^{1}$ (D, Érica Cardoso Moncayo ${ }^{1}$ (D),

Melissa Amanda Lourenço ${ }^{1}$ (D), Ruth Bernarda Riveros Jeneral ${ }^{1}$

\begin{abstract}
RESUMO
Introdução: A finalidade da assistência pré-natal é garantir o desenvolvimento da gestação, possibilitando o parto de um recém-nascido sadio, sem complicações para a saúde materna. Objetivo: Identificar e divulgar a percepção das puérperas que realizaram o pré-natal nas Unidades Básicas de Saúde (UBS) da região Oeste do município de Sorocaba frente às consultas de pré-natal vivenciadas. Método: Estudo descritivo, exploratório, de abordagem qualitativa. A coleta de dados foi realizada por meio de entrevista oral, gravada, orientada por uma questão norteadora. Os depoimentos foram organizados segundo o Discurso do Sujeito Coletivo (DSC), analisados e interpretados por meio da análise de conteúdo, modalidade temática. Resultados: Os resultados apontaram que a maioria das entrevistadas relatou assistência de qualidade durante as consultas de pré-natal, totalizando o surgimento de nove Ideias Centrais (IC). Conclusão: A assistência adequada durante as consultas de pré-natal é fundamental para o cumprimento dos princípios norteados pelo Sistema Único de Saúde (SUS) que envolvem promoção, prevenção, recuperação e reabilitação da saúde.

Palavras-chave: cuidado pré-natal; período pós-parto; percepção; saúde da mulher; centros de saúde.
\end{abstract}

\begin{abstract}
Introduction: The purpose of prenatal care is to ensure the development of pregnancy, allowing the birth of a healthy newborn without complications for maternal health. Objective: This study aimed to identify and disseminate the perception of puerperal women who performed prenatal care in the basic health units in the western region of the city of Sorocaba, in face of the prenatal consultations. Method: This was a descriptive, exploratory study with a qualitative approach. Data collection was performed through an oral, recorded interview, based on a guiding question. The statements were organized according to the Discourse of the Collective Subject, analyzed and interpreted through content analysis, thematic modality. Results: The results revealed that the majority of respondents reported having received good care during prenatal consultations, totaling the emergence of nine Central Ideas (CI). Conclusion: Adequate assistance during prenatal care is essential for compliance with the principles guided by the Unified Health System (SUS), which involve promotion, prevention, recovery, and rehabilitation of health.
\end{abstract}

Keywords: prenatal care; postpartum period; perception; women's health; health centers.

\section{INTRODUÇÃO}

A gestação é um momento único na vida da mulher. O nascimento do bebê é uma experiência peculiar e merece assistência integral e holística por profissionais qualificados. ${ }^{1}$

Em 1984, o Ministério da Saúde elaborou o Programa de Assistência Integral à Saúde da Mulher (PAISM), desta- cando conceitos com os princípios que norteavam a política de saúde das mulheres. ${ }^{2}$

O PAISM inclui ações educativas, preventivas, de diagnóstico, tratamento e recuperação envolvendo a assistência à mulher na clínica ginecológica, no pré-natal, no parto e no puerpério, no climatério, em planejamento familiar, infecções

${ }^{1}$ Pontifícia Universidade Católica de São Paulo, Faculdade de Ciências Médicas e da Saúde - Sorocaba (SP), Brasil. Autora correspondente: Ruth Bernarda Riveros Jeneral - Pontifícia Universidade Católica de São Paulo, Faculdade de Ciências Médicas e da Saúde, Departamento de Enfermagem - Rua Joubert Wey, 290 - CEP: 18030-070 - Sorocaba (SP), Brasil.E-mail: adoru@terra.com.br

Recebido em 15/02/2019 - Aceito para publicação em 30/05/2019. 
sexualmente transmissíveis (ISTs), prevenção do câncer de colo uterino e de mama, além de outras necessidades identificadas a partir do perfil populacional das mulheres. ${ }^{2}$

Atualmente, a consulta de enfermagem é realizada na Atenção Básica com respaldo na Lei do Exercício Profissional e de acordo com a rotina institucional. Para a Organização Mundial da Saúde (OMS), a assistência pré-natal objetiva proteger a saúde da mulher na gravidez e no aleitamento; ensinar-lhe cuidados voltados para as crianças; e favorecer o parto normal. Para isso, é necessário que haja uma assistência adequada desde o início da gestação por profissionais da saúde, como o(a) enfermeiro(a). ${ }^{3}$

A consulta ao pré-natal deve ser iniciada o mais cedo possível, e o profissional que assiste pode ser tanto o médico quanto o enfermeiro. A consulta exige a prática de acolhimento para a gestante e seu acompanhante. Dessa forma, devem ser acolhidas e esclarecidas as queixas, as dúvidas e as ansiedades, motivando o "desejo de voltar" ou a aceitação ao programa. ${ }^{4}$

A finalidade da assistência pré-natal é garantir o desenvolvimento da gestação, possibilitando o parto de um recém-nascido (RN) sadio, sem repercussão para a saúde materna, até mesmo se referindo aos aspectos psicossociais e às atividades educativas e preventivas. O principal indicador do prognóstico ao nascimento é a adesão à assistência pré-natal. As precauções assistenciais no primeiro trimestre gestacional são usadas como parâmetro de melhor qualidade dos cuidados maternos. ${ }^{5}$

Segundo a OMS, o número apropriado de consultas pré-natais é igual ou superior a seis. Pode ser que, mesmo com um número mais reduzido de consultas - porém com maior ênfase para o conteúdo de cada uma delas - , em casos de pacientes de baixo risco, não haja aumento de resultados perinatais adversos. Atenção especial deverá ser dada às grávidas com maiores riscos, que deverão ser encaminhadas a serviços específicos. $^{5}$

As consultas deverão ser mensais até a $28^{\mathrm{a}}$ semana, quinzenais entre 28 e 36 semanas e semanais até o termo. Quando o parto não acontece até a $41^{\mathrm{a}}$ semana, é preciso conduzir a gestante para a verificação do bem-estar fetal, envolvendo avaliação da quantidade do líquido amniótico e acompanhamento cardíaco fetal. ${ }^{6}$

Na primeira consulta pré-natal, o profissional de saúde necessitará: investigar a história clínica da gestante; constatar os antecedentes familiares, os antecedentes pessoais, os antecedentes ginecológicos, os dados sobre sexualidade, os antecedentes obstétricos; buscar informações da gestação atual; efetuar o exame físico geral e específico; e solicitar os exames laboratoriais preconizados. ${ }^{6}$

Considerando a necessidade de garantir atenção pré-natal e diminuição das altas taxas de morbimortalidade materna e perinatal, o Ministério da Saúde (MS) criou o Programa de Humanização do Pré-Natal e Nascimento (PHPN/2000) por meio da Portaria/GM n ${ }^{\circ} 569$, o qual propõe ratificar a melhora no acesso, na cobertura e na qualidade da assistência pré-natal, assim como do acompanhamento durante o parto e o puerpério, procurando uma atenção integral, qualificada e humanizada. $^{7}$

Nesse contexto, compreendemos a importância de identificar e divulgar a percepção das puérperas em relação ao atendimento recebido durante as consultas de pré-natal, visto que ainda há maternidades marcadas pela falta de atendimento adequado, pela ausência de estrutura e de preparo dos profissionais, bem como assistências de má qualidade, acarretando no aumento da morbimortalidade fetal e materna. Entende-se, então, que o aprofundamento da temática pode contribuir para a disseminação de uma melhor assistência.

\section{OBJETIVO}

Identificar a percepção das puérperas provenientes de Unidades Básicas de Saúde (UBS) que fazem parte da Regional de Saúde Oeste do Município de Sorocaba, atendidas no Alojamento Conjunto (AC) do Hospital Santa Lucinda (HSL), em relação à assistência recebida durante o pré-natal.

\section{MÉTODO}

Trata-se de uma pesquisa descritiva com análise qualitativa que incluiu 15 puérperas provenientes de UBS da regional oeste do município de Sorocaba.

Os dados foram obtidos por meio de entrevista gravada em áudio durante a estadia da puérpera no AC do HSL, após prévia autorização da mulher, com assinatura no Termo de Consentimento Livre e Esclarecido (TCLE). A questão norteadora foi: "Como você se sentiu durante as consultas de pré-natal?".

Para organização e análise dos dados foi utilizada a técnica do Discurso do Sujeito Coletivo (DSC), "que visa dar luz ao conjunto de individualidades semânticas componentes do imaginário social. É, em suma, uma forma destinada a fazer a coletividade falar indiretamente". ${ }^{8}$

"O quadro é composto de expressões-chave $(\mathrm{ECH})$ que são as partes ou fragmentos das falas das entrevistas, contínuos ou descontínuos, os quais vão expressar a essência do discurso ou a teoria subjacente" ${ }^{8,9} \mathrm{Na}$ seleção do material, buscou-se depurar o que é irrelevante e secundário, mantendo apenas o essencial nos discursos analisados, além de facilitar a construção deles. Nessa análise qualitativa foram seguidos os passos propostos por Lefévre e Lefévre. ${ }^{9}$ Como critérios de inclusão, foram consideradas puérperas de qualquer idade, em boas condições de comunicação e compreensão e que não apresentaram alguma condição patológica no puerpério; e como critérios de exclusão, puérperas que se recusaram a participar da pesquisa; que não estavam em boas condições pós-parto, em razão de óbito fetal (OF) e natimorto; ou que não estivessem em boas condições puerperais.

A pesquisa foi submetida à apreciação e à aprovação do Comitê de Ética em Pesquisa (CEP) da Pontifícia Universidade Católica de São Paulo (PUC-SP), sob número CAEE 67509617.0.0000.5373. 


\section{RESULTADOS}

\section{Caracterização dos participantes}

A amostra deste estudo foi composta de 15 puérperas que realizaram as consultas pré-natais nas 11 UBS pertencentes à Regional de Saúde Oeste de Sorocaba. As Tabelas 1, 2 e 3 apresentam algumas características do perfil dessas entrevistadas.

Os discursos do sujeito coletivo (DSC) representativos das Ideias Centrais (IC) apresentam-se por meio da questão norteadora da seguinte maneira: "Como você se sentiu durante as consultas de pré-natal?".

IC 1 - Boa assistência dos profissionais envolvidos no pré-natal

DSC 1 - puérpera (P)1, P2, P3, P4, P5, P6, P8, P9, P10, P11, P12, P13, P14, P15

Durante o pré-natal fui bem atendida e acolhida pelo médico, estagiários de medicina e pela enfermeira [...]. A minha primeira consulta foi com a enfermeira. Eles foram atenciosos, esclareceram todas as minhas dúvidas, pediram todos os exames e prescreveram as medicações [...]. $O$ atendimento na UBS é rápido, de fácil acesso, quando fui para a policlínica também fui bem atendida pelos profissionais.

Tabela 1. Características sociodemográficas das puérperas entrevistadas na maternidade do Hospital Santa Lucinda (Sorocaba, São Paulo, 2017).

$\begin{array}{lcc}\text { Variáveis } & \text { Puérperas entrevistadas } & \% \\ \text { Idade (anos) } & & \\ <20 & 3 & 20 \\ 20-30 & 8 & 53,3 \\ 31-35 & 3 & 20 \\ >35 & 1 & 6,6 \\ \text { Escolaridade } & & \\ \text { EFC } & 1 & 6,6 \\ \text { EFI } & 2 & 13,3 \\ \text { EMC } & 7 & 46,6 \\ \text { EMI } & 2 & 13,3 \\ \text { ESC } & 2 & 13,3 \\ \text { ESI } & 1 & 6,6 \\ \text { Total } & 15 & \end{array}$

EFC: ensino fundamental completo; EFI: ensino fundamental incompleto; EMC: ensino médio completo; EMI: ensino médio incompleto; ESC: ensino superior completo; ESI: ensino superior incompleto.
IC 2 - Encaminhamentos para pré-natal de alto risco

DSC 2 - P3, P4, P7, P12, P15

"Iniciei o pré-natal na UBS. Logo na primeira consulta com o médico fui transferida para gestação de alto risco devido a asma, sangramento, plaquetopenia, diabetes e pressão alta."

\section{IC 3 - Participação em grupos de gestantes}

DSC 3 - P3, P5, P10, P13, P14

A UBS tinha grupo de gestantes, que era muito bom, eu gostava do curso, falava de tudo, desde a gravidez até o parto. A equipe multiprofissional orientava

Tabela 2. Características obstétricas das puérperas entrevistadas na maternidade do Hospital Santa Lucinda.

\begin{tabular}{|c|c|c|}
\hline Variáveis & Puérperas entrevistadas & $\%$ \\
\hline \multicolumn{3}{|c|}{ Idade gestacional (semanas) } \\
\hline$<37$ & 1 & 6,6 \\
\hline $37-38$ & 4 & 26,6 \\
\hline $39-42$ & 10 & 66,6 \\
\hline \multicolumn{3}{|l|}{ Paridade } \\
\hline 1 & 8 & 53,3 \\
\hline $2-4$ & 7 & 46,6 \\
\hline \multicolumn{3}{|c|}{ Gestação (filhos) } \\
\hline $2-3$ & 11 & 73,3 \\
\hline $4-5$ & 4 & 26,6 \\
\hline \multicolumn{3}{|c|}{ Abortamento } \\
\hline Sim & 1 & 6,6 \\
\hline Não & 14 & 93,3 \\
\hline Total & 15 & \\
\hline
\end{tabular}

Tabela 3. Número de consultas realizadas pelo enfermeiro e pelo médico referidos pelas puérperas entrevistadas na maternidade do Hospital Santa Lucinda.

\begin{tabular}{ccc} 
Consultas & $\mathbf{N}^{\mathbf{0}}$ de consultas & $\%$ \\
Enfermeiro & & \\
Nenhuma & - & - \\
$1-6$ & 2 & 13,3 \\
$>6$ & 11 & 73,3 \\
Médico & & \\
$5-8$ & 7 & 46,6 \\
$9-11$ & 5 & 33,3 \\
12 & 3 & 20,0 \\
Total & 15 & \\
\hline
\end{tabular}


sobre os métodos não farmacológicos para alivio da dor, [...] amamentação, cuidados com o bebê, vacinas, planejamento familiar, tipos de parto, e no final mostravam vídeos [...]. Aprendi muito.

IC 4 - Falta de grupo de gestantes e palestras DSC 4 - P6, P9

Na UBS onde eu fazia o pré-natal não tinha grupo de gestantes, não recebi orientações [...], só tinha um informativo dizendo que poderia ir conhecer a maternidade do hospital [...]. A falta do grupo de gestantes não fez diferença para mim, já que a minha mãe é enfermeira, mas para as pessoas que não têm conhecimento o grupo é fundamental.

IC 5 - Não há diferença no atendimento do médico e do enfermeiro

DSC 5 - P3, P9

Fui bem atendida mesmo quando houve falta de médico [...]. Nas consultas que passei com a enfermeira tive um ótimo atendimento, ela explicava tudo [...]. Percebi pouca diferença no atendimento entre médico e enfermeiro, ambos esclareceram todas as minhas dúvidas.

IC 6-Má assistência por parte dos profissionais de saúde

DSC 6 - P4, P6, P8, P10, P12, P14, P15

"A médica que me atendeu não era boa, ainda bem que eu troquei [...]. A outra médica era estúpida, nem me examinava [...]. A enfermeira era bruta, também não examinava, era mal-educada, as consultas eram rápidas, só marcava consulta com o médico e apenas aferia a pressão quando eu pedia [...]. Tive descolamento de placenta e ela não se importou. Durante a consulta de alto risco também não fui bem atendida, o médico não era atencioso, não examinava, o USG [ultrassonografia] demorava para ser feito, não tirava minhas dúvidas [...]. Só fazia o básico e cumpria horário, às vezes nem colocava a mão em mim."

IC 7 - Falta de orientações na consulta de pré-natal

DSC 7 - P4, P6, P7, P11, P12

"Na UBS e na gestação de alto risco não tive nenhuma orientação do médico sobre parto, sinais de par- to, cuidados com o bebê [...], mas aqui no hospital me orientaram, desde quando o bebê nasceu."

\section{IC 8 - Falta de consulta com o enfermeiro DSC 8 - P4, P6}

\begin{abstract}
"Não passei em nenhuma consulta com o enfermeiro [...]. Quando descobri a gravidez já estava de quatro meses e fui encaminhada para a gestação de alto risco, recebi orientação do médico de que não precisava mais voltar no posto."
\end{abstract}

\section{IC 9 - Preferência pelo atendimento médico do que pela enfermagem}

DSC $9-\mathrm{P} 13$

"Na UBS que iniciei o pré-natal só tinha enfermeiro, então mudei de postinho, pois prefiro ouvir opiniões e orientações de médico do que de enfermeiro."

\section{DISCUSSÃO}

Neste estudo, foram entrevistadas 15 puérperas que realizaram as consultas pré-natais nas UBS pertencentes à região oeste de Sorocaba. Os dados qualitativos foram obtidos por meio de entrevistas gravadas em áudios e transcritas e analisadas por meio do método do DSC.

Em relação à caracterização das puérperas entrevistadas, prevaleceram as adultas jovens, com idade entre $20 \mathrm{e}$ 30 anos $(53,3 \%)$.

De acordo com a escolaridade, constatou-se que das 15 puérperas entrevistadas, sete concluíram o ensino médio (46,6\%), além de nenhum caso de entrevistada sem escolaridade.

Em relação às variáveis obstétricas, verificou-se que dez puérperas $(66,6 \%)$ tiveram seus bebês a termo e apenas uma teve seu bebê prematuro, com 36 semanas de gestação $(6,6 \%)$.

Quanto ao atendimento recebido durante as consultas de pré-natal, a maioria das puérperas entrevistadas referiu que recebeu boa assistência dos profissionais envolvidos. Em outros estudos, ${ }^{10,11}$ a maior parte das puérperas também relatou boa assistência e satisfação com o atendimento dos profissionais de saúde. Referiram que foram orientadas; acolhidas com escuta ativa, de maneira que se sentiram seguras; e que suas queixas, anseios e dúvidas foram esclarecidos nas consultas, nas palestras e nas aulas sobre prevenção de doenças, nos procedimentos referentes ao diagnóstico e ao tratamento ofertado no pré-natal, como a execução de exames e a disponibilidade de vacinas - uma boa assistência que permite um vínculo mais próximo entre enfermeiro e paciente.

Ainda em relação à qualidade da assistência, verificou-se que algumas entrevistadas expressaram a não efetividade do pré-natal e em seus relatos manifestaram insatisfação com o atendimento recebido, com a sensação de não 
estarem realizando o pré-natal, pois chegavam à maternidade desinformadas, necessitando de orientações e cuidados durante o pré-natal. ${ }^{10}$

Em conformidade com os achados encontrados na literatura, ${ }^{10}$ percebe-se nas falas das entrevistadas que os médicos na unidade básica, durante o pré-natal, não ofereciam um bom atendimento e não esclareciam as dúvidas das gestantes. Uma das entrevistadas relatou que os médicos não examinavam as gestantes e a enfermeira era indelicada, não dava a atenção necessária, realizava consultas rápidas, não questionava e nem examinava a paciente, aferia somente a pressão arterial (PA) e marcava a próxima consulta com o médico.

Em relação à falta de consultas de enfermagem no pré-natal, não se constatou na literatura que isso ocorra com frequência, pois todas as pesquisas confirmam que as gestantes passam pelo menos em uma consulta com o enfermeiro e que esse profissional é fundamental na assistência durante o pré-natal. Nesta pesquisa, os dois casos que relataram não terem ocorrido consultas de pré-natal com enfermeiro se justificam, pois uma das entrevistadas foi encaminhada diretamente para o pré-natal de alto risco em razão de plaquetopenia e a outra informou que descobriu a gravidez tardiamente, com quatro meses de gestação, sendo então encaminhada pela enfermeira para passar diretamente com o médico em razão de gestação de alto risco e gestação tardia.

Em relação à participação das mulheres em grupos de gestantes, vemos que a Lei Orgânica da Saúde nº 8.080 dispõe que a atenção à saúde pelo Sistema Único de Saúde (SUS) deve incluir as ações assistenciais ou curativas e, preferencialmente, as práticas de promoção da saúde e prevenção de doenças. $\mathrm{O}$ atendimento básico é representado pela assistência de maneira preventiva e promocional. ${ }^{12,13}$

O Ministério da Saúde ${ }^{14}$ preconiza que no decorrer do pré-natal a gestante deve receber orientações especialmente sobre os assuntos: importância do pré-natal; processo de gravidez; mudanças corporais e emocionais durante a gravidez; trabalho de parto; parto e puerpério; cuidados com o RN; e amamentação. É fundamental também o envolvimento do pai no pré-natal, sendo necessário estimular sua participação no período de rotinas de consulta e de grupo de gestantes para o treinamento dos futuros pais para o momento do parto. ${ }^{14}$

Segundo Santos et al., a educação em saúde:

Trata-se de um recurso por meio do qual o conhecimento cientificamente produzido no campo da saúde, intermediado pelos profissionais de saúde, atinge a vida cotidiana das pessoas, uma vez que a compreensão dos condicionantes do processo saúde-doença oferece subsídios para a adoção de novos hábitos e condutas de saúde. ${ }^{15}$

Por intermédio das atuações educativas, o enfermeiro deve trabalhar, no individual e no coletivo, os itens relacionados ao parto, à amamentação, à vacinação, ao crescimento e ao desenvolvimento do bebê e os cuidados com o RN. ${ }^{15}$

Em outro estudo, constatou-se que a sala de espera é o local ideal para orientações, pois os profissionais da área da saúde têm a chance de desenvolver atividades educativas. As palestras foram essenciais para promover espaço para que as gestantes se sentissem acolhidas, protegidas e pudessem expressar suas dúvidas, anseios, medos e dificuldades e desenvolver ações educativas não comuns na sala de espera. ${ }^{15}$

Dessa forma, mediante a construção em conjunto, as gestantes tiveram a oportunidade de se expressar, aprender, criar, compartilhar e refletir sobre as competências e as barreiras associadas à saúde integral individual e coletiva. ${ }^{15}$

Diante dos resultados do presente estudo, em concordância com os achados de outros autores, citados anteriormente, das 15 puérperas entrevistadas, cinco referiram participação em grupos de gestantes no decorrer do pré-natal. No discurso de uma das gestantes que participou do grupo neste estudo, percebeu-se que a educação em saúde foi realizada pela equipe multiprofissional de forma acolhedora, empática e esclarecedora, utilizando-se de métodos tecnológicos e lúdicos, favorecendo uma assistência de qualidade, integral, holística e humanizada.

Neste estudo, observou-se também que existem alguns problemas que dificultam a participação das gestantes. Duas delas relataram que tinham compromisso de trabalho nos dias e horários pré-estabelecidos pela Unidade de Saúde. Assim, é necessário que o planejamento, a elaboração e a organização dos grupos de gestantes sejam pactuados com elas para que todas possam participar efetivamente. Outro ponto foi a ausência dos grupos de gestantes em algumas Unidades de Saúde. Nos relatos das puérperas, algumas referiram que sentiram necessidade de mais orientações e de um local onde pudessem esclarecer as dúvidas relacionadas à gestação, ao parto, à amamentação e aos cuidados com o RN.

Em relação à falta de grupos de gestantes ou palestras nas Unidades Básicas, constatou-se que são frequentes ações e procedimentos individuais e usuais relacionados aos cuidados em saúde em contraponto à ação coletiva e grupal. Os profissionais, em suas ações, preferem os atendimentos individuais relacionados aos procedimentos tradicionais, possivelmente por falta de instrumentos e conhecimentos. ${ }^{16}$

Segundo Rios e Vieira ${ }^{17}$ e Ceron et al. ${ }^{7}$, as gestantes relataram o descontentamento em relação à escassez de informações e orientações relacionadas ao pré-natal, ao parto, ao puerpério e ao cuidado com o $\mathrm{RN}$ e à falta de esclarecimentos nas Unidades de Saúde.

Os resultados desta pesquisa são semelhantes aos achados dos autores citados anteriormente.

Em relação ao encaminhamento para o pré-natal de alto risco, o SUS oferece, em suas diretrizes, uma assistência integral às gestantes, levando em consideração a rede de serviços do sistema de referência e de contrarreferência.

Neste estudo, constatou-se que as gestantes de alto risco, na maioria das vezes, necessitam de técnicas mais individualizadas, acompanhamento diferenciado e próximo, exames periódicos e rápidos, pois as complicações levam a risco maior, tanto para a mãe quanto para o bebê. ${ }^{18}$

De acordo com os achados deste trabalho, as gestantes que foram encaminhadas para o pré-natal de alto risco 
referiram bom atendimento na assistência especializada, recebendo orientações específicas para a complicação que apresentavam, esclarecendo dúvidas, tranquilizando os próprios medos e anseios, queixando-se apenas da demora para a realização de exames periódicos, como o USG obstétrico e exames laboratoriais.

Em relação à preferência do atendimento pelo médico do que pela enfermeira, observou-se que o tempo de conversa com o médico muitas vezes é insuficiente, impossibilitando a criação de um vínculo eficaz. Já as consultas com os enfermeiros eram mais ativas, facilitando vínculo cliente-enfermeiro. ${ }^{19}$

Neste estudo, apenas uma puérpera referiu preferência pelo atendimento médico e relatou mudança de unidade básica em razão da falta deste, reafirmando a preferência por opiniões e orientações médicas. Esse relato é comprovado em outro estudo, ${ }^{20}$ que constata que a figura do enfermeiro pode causar receios e sentimento de insegurança nas gestantes, que é decorrente de uma idealização cultural voltada para o modelo biomédico.

Em relação à diferença no atendimento do médico e do enfermeiro, verificou-se neste estudo que as enfermeiras desenvolvem ações, iniciativas, habilidades e competências focadas no cuidar integral e holístico das grávidas durante o pré-natal, centradas nas ações de prevenção de doenças, promoção e recuperação da saúde. As gestantes expressaram satisfação com a assistência prestada pela enfermeira no pré-natal, ao referir ações de cuidado que promovem a escuta e o acolhimento em relação ao binômio mãe/filho, desconstruindo um modelo de cuidar frio e impessoal ${ }^{21}$ — visto em alguns discursos como falta de orientações médicas em consulta de pré-natal (IC 7) e de como o grupo de gestantes (IC 3) desenvolvido por enfermeiros era necessário para elas, pois era o local onde esclareciam suas dúvidas.

O reconhecimento das enfermeiras ocorre em razão dos valores sociais atribuídos às ações e às práticas realizadas por esse profissional em razão da escuta ativa e da qualidade do atendimento. Esse cuidado assegura a compreensão, a empatia e a formação de vínculo entre profissional e paciente, confiança e práticas voltadas para o bem-estar das pacientes. Assim, a assistência desenvolvida pelos profissionais enfermeiros no pré-natal proporciona maior liberdade das gestantes, tornando a consulta mais eficaz.

Em uma das falas no estudo de Barbosa, a cliente refere que o médico é rápido, não tira as dúvidas e, quando questionado, não examina direito e não faz perguntas, além de informar que a enfermeira supera na atenção e que os médicos nem sempre conseguem fazê-lo por falta de tempo. ${ }^{22}$

Nesta pesquisa, identificou-se que a maior parte das puérperas se sentiu segura, acolhida em realizar a consulta com o enfermeiro, e referiu bom atendimento, pois "tiveram liberdade de perguntar e esclarecer todas as dúvidas". Outras relataram não haver diferença no atendimento entre médico e enfermeiro, pois "quando houve falta de médico na unidade, a enfermeira realizou a consulta de pré-natal [...] e duas puérperas referiram que ambos os profissionais exerceram a mesma função".
Constatou-se, também, a boa assistência por parte dos profissionais envolvidos no pré-natal por meio dos DSCs P1, P2, P3, P4, P5, P6, P8, P9, P10, P11, P12, P13, P14 e $\mathrm{P} 15$. O DSC que corroborou a "boa assistência" por parte dos profissionais foi a ideia mais compartilhada entre os entrevistados em relação à questão, resultando no seguinte discurso:

\begin{abstract}
Durante o pré-natal fui bem atendida e acolhida pelo médico, estagiários de medicina e pela enfermeira [...]. A minha primeira consulta foi com a enfermeira. Eles foram atenciosos, esclareceram todas as minhas dúvidas, pediram todos os exames e prescreveram as medicações [...]. O atendimento na UBS é rápido, de fácil acesso, quando fui para a policlínica também fui bem atendida pelos profissionais.
\end{abstract}

\section{CONCLUSÃO}

$\mathrm{O}$ estudo permitiu identificar, pontuar e conhecer a percepção das puérperas em relação ao atendimento recebido na UBS durante a consulta de pré-natal. Isso demonstra que a atenção primária está conseguindo cumprir seus deveres, norteados pelos princípios do SUS, que envolvem promoção, prevenção, recuperação e reabilitação da saúde.

Apesar de não fazer parte da pesquisa e não ter relação com os objetivos deste trabalho, foi interessante o relato das puérperas entrevistadas sobre o pré-parto, o parto e o puerpério. O que chamou a atenção foi o fato de todas elas referirem boa assistência, atenção, escuta ativa, vínculo, orientações, esclarecimento de dúvidas, afeto e acolhimento por parte de todos os profissionais envolvidos durante o período do pré-parto, do parto e do puerpério na área hospitalar, médicos, residentes, estudantes de enfermagem e medicina, fonoaudiólogos, nutricionistas e, principalmente, toda a equipe de enfermagem, como afirmam nos DSCs citados anteriormente.

É papel do enfermeiro como integrante da equipe durante o decorrer do pré-natal e da fase puerperal esclarecer as dúvidas, auxiliar e preparar as gestantes para essa nova etapa. $\mathrm{O}$ enfermeiro precisa atuar ativamente não só em relação às transformações da gestação e da chegada do bebê, mas também na vivência da mulher nessa nova fase da vida.

Acredita-se, então, que o conhecimento produzido não se esgota neste estudo, necessitando ser ampliado. A realização de novas pesquisas relacionadas a gestantes e ao bom atendimento durante o pré-natal é de grande importância, por isso é necessário refletir e avaliar as condições para se ter um bom atendimento durante as consultas de pré-natal, levando em consideração os benefícios para mãe e filho.

\section{REFERÊNCIAS}

1. Vieira SM, Bock LF, Zocche DA, Pessota CU. Percepção das puérperas sobre a assistência prestada pela equipe de saúde no pré-natal. Texto Contexto Enferm. 2011;20(n. esp.):255-62. doi: http://dx.doi. org/10.1590/S0104-07072011000500032 
2. Brasil. Ministério da Saúde. Secretaria de Atenção à Saúde. Departamento de Ações Programáticas Estratégicas. Política Nacional de Atenção Integral à Saúde da Mulher. Princípios e Diretrizes. Brasília: Ministério da Saúde; 2004.

3. Marques RG, Prado SRLA. Consulta de enfermagem no pré-natal. Rev Enferm UNISA. 2004;5:33-6.

4. São Paulo (Governo do Estado). Secretaria de Estado da Saúde de São Paulo. Manual técnico do pré-natal e puerpério: atenção a gestante e a puérpera no SUS-SP. São Paulo: Secretaria de Estado da Saúde; 2010.

5. Brasil. Ministério da Saúde. Secretaria de Atenção à Saúde. Departamento de Atenção Básica. Atenção ao pré-natal de baixo risco. Brasília: Ministério da Saúde; 2012.

6. Araújo SM, Silva MED, Moraes RC, Alves DS. A importância do pré-natal e a assistência de enfermagem. Veredas. 2010;3(2):61-7.

7. Ceron MI, Barbieri A, Fonseca LM, Fedosse E. Assistência pré-natal na percepção de puérperas provenientes de diferentes serviços de Saúde. Rev CEFAC. 2013;15(3):653-62. doi: http://dx.doi. org/10.1590/S1516-18462012005000081

8. Lefévre F, Lefévre AMC, Teixeira JJV. O Discurso do Sujeito Coletivo: uma nova abordagem metodológica em pesquisa qualitativa. Caxias do Sul: EDUCS; 2000.

9. Lefévre F, Lefévre F. O Discurso do Sujeito Coletivo: um novo enfoque em pesquisa qualitativa (desdobramentos). Caxias do Sul: EDUCS; 2005.

10. Castro ME, Moura MAV, Silva LMS. Qualidade da assistência pré-natal: uma perspectiva das puérperas egressas. Rev Rene. 2010;11:72-81.

11. Afonso JA, Afonso KKA, Jones KM. Percepção das gestantes frente ao pré-natal prestado pelo enfermeiro. Rev Bras Pesq Ciênc Saúde. 2015;2(1):22-6.

12. Sousa AJCQ, Mendonça AEO, Torres GV. Atuação do enfermeiro no pré-natal de baixo risco em uma unidade básica de saúde. Carpe Diem. 2012;10(1):1-15.

13. Duarte SJH, Andrade SMO. O significado do pré-natal para mulheres grávidas: uma experiência no município de Campo Grande, Brasil. Saúde Soc.
2008;17(2):132-9. doi: http://dx.doi.org/10.1590/ S0104-12902008000200013

14. Brasil. Ministério da Saúde. Guia prático do Programa Saúde da Família. Brasília: Ministério da Saúde; $2001.67 \mathrm{p}$.

15. Santos DS, Andrade ALA, Lima BSS, Silva YN. Sala de espera para gestantes: uma Estratégia de Educação em Saúde. Rev Bras Educ Med. 2012;36(1 Supl. 2):62-7. doi: http://dx.doi.org/10.1590/S0100-55022012000300010

16. Silvestrini MS, Cardoso MLM, Rego BR. Desafios na construção de um grupo de gestantes na Unidade de Saúde da Família. Cad Ter Ocup UFSCar. 2014;22(3):603-7. doi: https://doi.org/10.4322/cto.2014.082

17. Rios CTF, Vieira NFC. Ações educativas no pré-natal: reflexão sobre a consulta de enfermagem como um espaço para educação em saúde. Ciênc Saúde Coletiva. 2007;12(2):477-86. doi: http://dx.doi.org/10.1590/ S1413-81232007000200024

18. Buchabqui JA, Capp E, Ferreira J. Adequação dos encaminhamentos de gestações de alto-risco na Rede Básica de Atenção à Saúde de Porto Alegre, Rio Grande do Sul, Brasil. Rev Bras Saúde Matern. 2006;6(1):23-9. http://dx.doi.org/10.1590/S1519-38292006000100003

19. Oliveira CB, Ramos MC. O grau de satisfação da usuária gestante na assistência pré-natal nas unidades de saúde da família no município de Vitória. Cad Saúde Coletiva. 2007;15(2):241-56.

20. Barreto CN, Wilhelm LA, Silva SC, Alves CN, Cremonese L, Ressel LB. O Sistema Único de Saúde que dá certo: ações de humanização no pré-natal. Rev Gaúcha Enferm. 2015;36(esp):168-76. DOI: http:// dx.doi.org/10.1590/1983-1447.2015.esp.56769

21. Moreira MA, Carvalho LL, Ribeiro OS. Percepção de gestantes sobre a atuação da enfermeira na assistência pré-natal: estudo analítico. Arq Ciênc Saúde. 2016;23(1):78-82. DOI: https://doi.org/10.17696/23183691.23.1.2016.217

22. Barbosa TLA, Gomes LMX, Dias OV. O pré-natal realizado pelo enfermeiro: a satisfação das gestantes. Cogitare Enferm. 2011;16(1):29-35. DOI: https://doi. org/10.5380/ce.v16i1.21108

\section{Como citar este artigo:}

Kawatsu MM, Moncayo EC, Lourenço MA, Jeneral RBR. Percepção das puérperas em relação ao atendimento recebido na unidade básica de saúde durante a consulta de pré-natal. Rev Fac Ciênc Méd Sorocaba. 2019;21(4):170-6. DOI: $10.23925 / 1984-4840.2019 v 21 \mathrm{i} 4 \mathrm{a} 6$ 